\title{
MINERAL TRIOXIDE AGGREGATE VS CALCIUM ENRICHED MIXTURE MOLAR PULPOTOMY: A SYSTEMATIC REVIEW AND META-ANALYSIS
}

\author{
Marwa El-Sayed Sharaan* and Mohamed Ibrahim Rabie *
}

\begin{abstract}
Objectives: The aim of the current systematic review /meta-analysis was to perform a comparison of the outcomes of Calcium Enriched Mixture (CEM) and Mineral Trioxide Aggregate (MTA) as agents for pulpotomy in primary and permanent molars. The proposed PICO question was "Whenever there is vital pulp exposure in primary and permanent molars, what are the treatment outcomes of CEM compared to MTA pulpotomy regarding clinical and radiographic success?"

Methods: We looked for published randomized clinical trials (RCTs) of the at least 12-month period; the search covered papers published up to July 2017 in PubMed (Medline) and SpringerLink databases. Meta-analysis was performed using R program 3.3.3 with specialized meta-analysis packages, namely rmeta, metaphor, and RGtk2. Data was reformulated dichotomously (success or other [healing, failure, or missing]) for the selected studies. The fixed effect model was tested, OR (Odds ratio), RR (Risk ratio), and 95\% CI (Confidence interval) were calculated using the formulated raw dichotomous data of the selected studies. The heterogeneity among studies was assessed using standard chi- square test and Woolf's test. Mantel-Haenszel fixed-effect method was used to compute OR for the fixed model. The level of significance was at 0.05.
\end{abstract}

Results: Four hundred forty-nine articles were gathered. Two reviewers screened the articles, and three RCTs were included in the study. After the assessment, three RCTs were suitable for meta-analysis tested by Mantel Haenszel method. Statistically, the insignificant difference was found between the success rate of CEM compared to MTA, with $\mathrm{OR}=0.92(95 \% \mathrm{CI}, 0.57-1.49)$. RR $=0.94$ (95\% CI, 0.66-1.35). Test for heterogeneity showed p-values $(0.4241,0.4455)$

Conclusions: Systematic review /meta-analysis of the chosen RCTs showed that CEM pulpotomy in human teeth presented insignificant but superior clinical and radiographic success compared to those treated with MTA. Good quality and homogeneity of the included RCTs were yielded.

KEY WORDS: Calcium Enriched Mixture, Meta-Analysis, Mineral Trioxide Aggregate, Molar pulpotomy, Systematic review.

\footnotetext{
* Lecturer in Endodontics, College of Dentistry, Suez Canal University
} 


\section{INTRODUCTION}

Whenever there was a carious vital pulp exposure, the clinician may decide to cover the exposed pulp by direct pulp capping, pulpotomy or to start a conventional root canal treatment ${ }^{(1)}$. Treatment modality depends on several factors, including factors related to patients such as medical history and age. In addition to tooth factors, for instance, whether it is a primary or permanent tooth ${ }^{(2)}$. Pulpotomy is documented for its favorable results in variously exposed pulps in primary molars ${ }^{(1,2)}$. This procedure can be defined as "the surgical removal of the coronal portion of the pulp and the placement of a therapeutic agent to preserve the health of the remaining vital tissues" ${ }^{(1,2)}$. In the permanent dentition, a pulpotomy is considered to be amongst the most frequently used emergency treatment methods to decrease pain for irreversible pulpitis ${ }^{(3)}$. The excellent outcome of root canal treatment is well established ${ }^{(3)}$. On the other hand, the procedure is time-consuming and complicated in some cases. However, a common different possible treatment choice might be extraction because of financial limitations in some parts of the world ${ }^{(4)}$. Currently, the use of conventional and straightforward pulpotomy techniques using one of the two biomaterials: [1] mineral trioxide aggregate (MTA) and [2] calcium enriched mixture (CEM) cement ${ }^{(4-18)}$ was recommended.

MTA has been introduced for pulpotomy in both primary and permanent molars. It has turned into a commonly used biomaterial for vital pulp treatment (VPT) ${ }^{(4-10)}$ because of its capability to generate hard-tissue formation in pulpotomy treatment ${ }^{(11,12)}$. A histological examination of human mature permanent molars with irreversible pulpitis, revealed complete dentinal bridge formation, pulp vitality, and absence of inflammation in all the cases ${ }^{(12,13)}$. Despite its excellent properties, it has been reported that MTA shows disadvantages including a non-predictable antimicrobial activity, challenging manage- ment, expanded setting time, and the most significant problem, especially in the developed countries, is the high price ${ }^{(4)}$.

CEM is a favorable hydrophilic tooth colored cement ${ }^{(9-11,14-19)}$. It is composed of different calcium compounds such as calcium silicate, sulfur tricalcium, calcium chloride, calcium phosphate, calcium oxide, calcium hydroxide and calcium carbonate ${ }^{(14)}$. It has several favorable biological and physical properties $^{(18)}$. Compared to MTA, it is characterized by its shorter setting time, good handling, better flowability and less film thickness ${ }^{(18)}$. It is also characterized by its sealing ability, biocompatibility, ability to form hydroxyapatite, ability to induce cementum ${ }^{(19)}$. CEM is also characterized by its efficient antimicrobial activity. It is widely used as a pulp capping agent, retrograde filling and in repairing root perforation ${ }^{(19)}$

Meta-analysis has been graded as the highest rank of evidence available to clinicians to guide the clinical dental practice ${ }^{(20)}$. Some authors compared the outcomes of CEM and MTA as agents for pulpotomy in primary and permanent molars, but there is no agreement which regimen is attributed to the superior results. Hence, the purpose of the present study is to conduct a meta-analysis comparing the results of CEM and MTA as agents for pulpotomy in primary and permanent molars.

\section{MATERIALS AND METHODS}

\section{PICO question}

Whenever there is vital pulp exposure in primary and permanent molars, what are the treatment outcomes of CEM compared to MTA pulpotomy regarding clinical and radiographic success?

\section{Protocol and registration}

This systematic review (SR) was undertaken using the recommended guidelines ${ }^{(21)}$. The present SR 
was performed according to the Preferred Reporting Items for Systematic Reviews and Meta-analyses (PRISMA) statement ${ }^{(22)}$. A study protocol was written and registered with the public registry of systematic reviews PROSPERO (CRD42017071664).

\section{Literature search}

MEDLINE ( by using Entrez PubMed search engine ) and Springer link databases were searched from inception until July 31, 2017, using the addition of the keywords pulpotomy, permanent teeth, primary teeth MTA, and CEM. Articles in the English language were the selected ones. RCTs performed on primary and permanent molars have been chosen.RCTs comparing CEM and MTA were recognized using the following enquiry: ("mineral trioxide aggregate" [Mesh] OR ("mineral trioxide" Field: Title/Abstract) AND ("calcium enriched mixture»[Mesh] OR («calcium enriched mixture» Field: Title/ abstract)". Searches were performed by two reviewers to identify relevant articles. References of all review articles were also checked manually. Grey literature was not searched.

\section{Inclusion and exclusion criteria}

The inclusion criteria were: (A) Randomized controlled trials which compared CEM to MTA pulpotomies; (B) in primary and permanent symptomatic/asymptomatic; (C) mature/ immature;(D) restorable teeth ; (E) with vital pulp exposure either by caries or trauma; $(\mathrm{F})$ the presence of bleeding upon exposure; $(\mathrm{G})$ reported quantitative clinical or radiographic outcomes; and $(\mathrm{H})$ follow up time of at least up to 12 months. Exclusion criteria were: (A) lack of randomization; (B)animal or in vitro studies; (C) absence of comparison between the treatment groups; (D) the article could not be found; (E) Only histological evaluation of CEM versus MTA; (F) Indirect/direct pulp capping procedure ; $(\mathrm{G}) \mathrm{Com}-$ ments, editorials, case reports, proceedings, and personal communications and $(\mathrm{H})$ Studies did not report a primary quantitative outcome were also excluded.

\section{Data extraction and quality assessment}

Before starting data mining, duplication was removed. Then the data was extracted and screened from the full texts by two reviewers using abstrackr. If there was no agreement, rechecking the text and discussion were done.The quality of selected studies was evaluated according to validity criteria (van Tulder list) ${ }^{23)}$ (Table 1). The validity of the chosen articles was ascertained by assessment by the reviewers. Whenever there was a disagreement, the meeting was held to solve it.

\section{Meta-analysis}

Meta-analysis was performed using $\mathrm{R}$ program 3.3.3 with specialized meta-analysis packages namely rmeta, metaphor, and RGtk2. Data was reformulated dichotomously (success or other [healing, failure, or missing]) for the selected studies. Fixed effect model was tested. OR (Odds ratio), RR (Risk ratio), and 95\% CI (Confidence interval) were calculated using the formulated raw dichotomous data of the selected studies. The heterogeneity among studies was assessed using standard chi- square test and Woolf's test. Mantel-Haenszel fixed-effect method was used to compute OR for the fixed model, respectively. The level of statistical significance was at 0.05 .

\section{RESULTS}

\section{Study selection and data summary}

Searches in Medline and SpringerLink databases displayed 449 English published studies.The search protocol is shown in Figure (1) . Three studies comparing CEM and MTA molar pulpotomy with a total of 504 teeth met inclusion criteria (Table 1). ${ }^{(8-10)}$. 


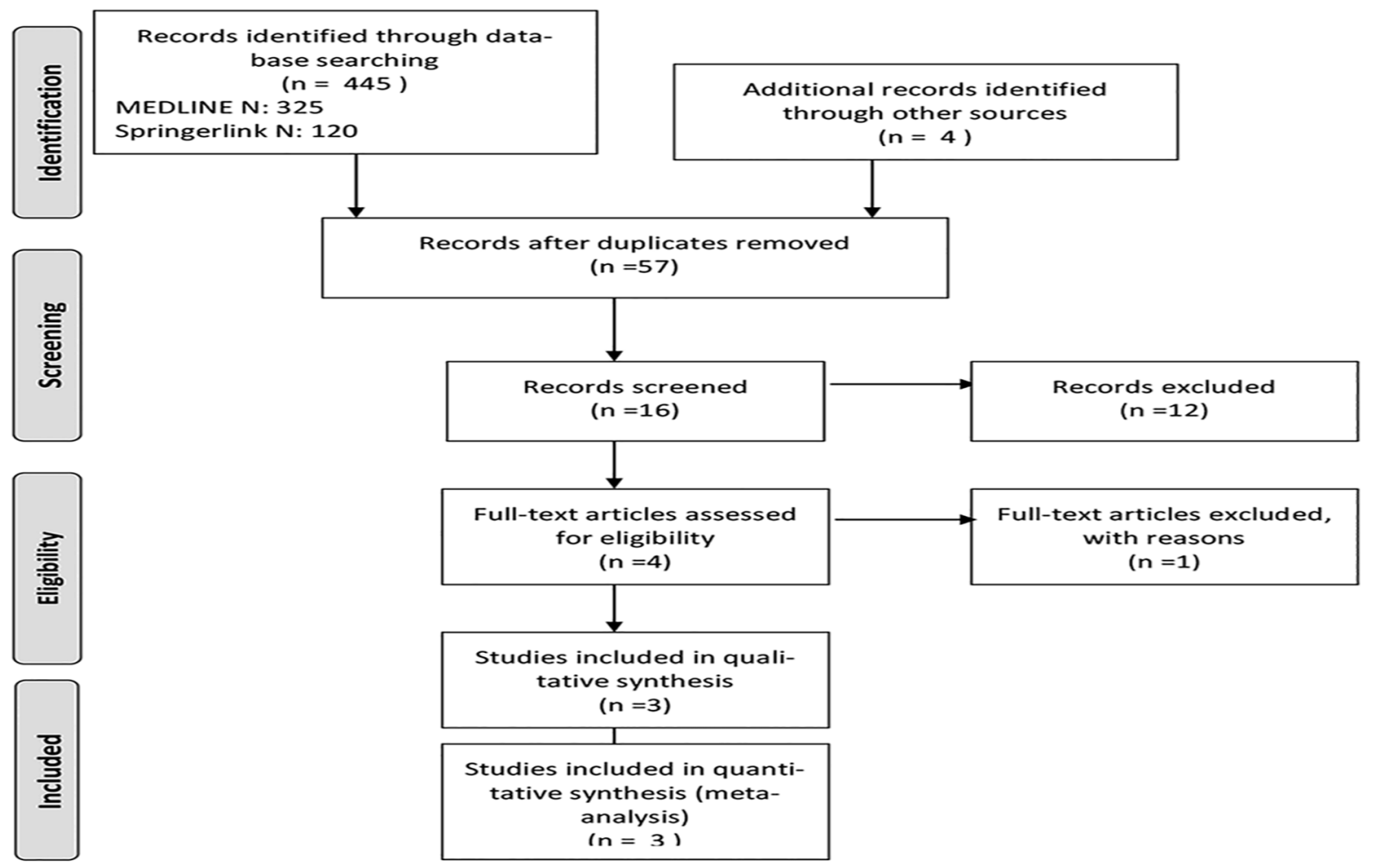

Fig. (1) Article assortment flow chart to the systematic review by PRISMA guidelines

TABLE (1) Inclusion/exclusion criteria of the three RCTs included in the meta-analysis

\begin{tabular}{|c|c|c|}
\hline Study & Inclusion criteria & Exclusion criteria \\
\hline $\begin{array}{l}\text { Nosrat et al. } \\
2013\end{array}$ & $\begin{array}{l}\text { Symptomatic / asymptomatic vital immature (radio- } \\
\text { graphically open apex) first permanent molars with } \\
\text { clinical carious exposure of the pulp and the presence } \\
\text { of bleeding upon exposure. }\end{array}$ & $\begin{array}{l}\text { Non-Restorable tooth; presence of related sinus tract; } \\
\text { soft tissue swelling / redness over peri-radicular tis- } \\
\text { sues; the presence of peri- and / or interradicular le- } \\
\text { sions; internal / external root resorption; pulp / canal } \\
\text { calcifications and excessive mobility (more than } 1 \mathrm{~mm} \\
\text { horizontally). }\end{array}$ \\
\hline $\begin{array}{l}\text { Asgary and } \\
\text { Eghbal } 2013\end{array}$ & $\begin{array}{l}\text { Impulsive pain for several seconds to a few hours with } \\
\text { extensive caries,; pain exacer- bated with cold and hot } \\
\text { fluids and/or; radiating pain and teeth had to respond } \\
\text { to cold vitality testing and bleeding from all canal ori- } \\
\text { fices had to be observed during access cavity prepara- } \\
\text { tion }\end{array}$ & $\begin{array}{l}\text { Active systemic disease; physical or mental disability } \\
\text { and/ or patients who were pregnant or nursing ; teeth } \\
\text { showed signs of (a) moderate/severe marginal peri- } \\
\text { odontitis; (b) were non-restorable; (c) had internal or } \\
\text { external root resorption; (d) had root canal calcifica- } \\
\text { tion; and (e) had associated abscesses. }\end{array}$ \\
\hline $\begin{array}{l}\text { Malekafzali et } \\
\text { al. } 2011\end{array}$ & $\begin{array}{l}\text { The existence of a minimum of two teeth with carious } \\
\text { exposure; Symptom-Free primary molars with a deep } \\
\text { caries lesion and presence of a vital pulp and restorable } \\
\text { crowns. }\end{array}$ & $\begin{array}{l}\text { Non-Carious exposures after treatment ; clinical and/or } \\
\text { radiographic sign or symptoms of pulp degeneration } \\
\text { (i.e. spontaneous pain, inter-radicular and/or periapi- } \\
\text { cal bone destruction, internal root resorption, excessive } \\
\text { bleeding from the root canal, tenderness to percussion, } \\
\text { swelling or acute/chronic apical abscess, pathologi- } \\
\text { cal mobility); active systemic disease and physical or } \\
\text { mental disability. }\end{array}$ \\
\hline
\end{tabular}




\section{Characteristics of included studies}

All studies were performed in Iran. Two studies were conducted in a university teaching hospital environment. Also, one of these was carried out in an Iranian Center for Endodontic Research.Furthermore, the third study was conducted at the Iranian Center for Endodontic Research, by different clinicians. Features of the provided experiments were displayed in Table 2.

TABLE (2) Characteristics of the RCTs included in meta-analysis

\begin{tabular}{|c|c|c|c|c|c|}
\hline Study/country (year) & $\begin{array}{c}\text { No. of } \\
\text { Participants }\end{array}$ & No. of teeth & Age range (yr) & Type of teeth & Lost to follow up \\
\hline $\begin{array}{c}\text { Nosrat et al. / Iran 2013 } \\
\text { Asgary and Eghbal/ } \\
\text { Iran 2013 }\end{array}$ & 51 & 51 & $7-10$ & Permanent molars & $\begin{array}{c}3 \text { after } 6 \mathrm{mth} \\
2 \text { after } 12 \mathrm{mth}\end{array}$ \\
\hline $\begin{array}{c}\text { Malekafzali et a./ Iran } \\
\text { 2011 }\end{array}$ & 40 & 413 & $9-65$ & Permanent molars & 67 after $12 \mathrm{mth}$ \\
\hline
\end{tabular}

\section{Methodological quality assessment of included studies}

Methodological quality was evaluated with the use of a scale developed and validated by van Tulder list. According to these criteria, study quality scores were 10 for the studies by Nosrat et al. and Asgary and Eghbal 2013, Malekafzali et al. scored 9 according to the van Tulder list. (Table 3).

TABLE (3) Methodological quality assessment of included studies (van Tulder list)

\begin{tabular}{|c|c|c|c|}
\hline & $\begin{array}{l}\text { Nosrat } \\
\text { et al. } 2013\end{array}$ & $\begin{array}{c}\text { Asgary and } \\
\text { Eghbal } \\
2013\end{array}$ & $\begin{array}{l}\text { Malekafzaki } \\
\text { et al. } 2011\end{array}$ \\
\hline Was an appropriate method of randomization performed? & Yes & Yes & Yes \\
\hline Was the treatment allocation concealed? & Yes & Yes & $\mathrm{N} / \mathrm{S}$ \\
\hline Was the care provider blinded? & $\mathrm{N} / \mathrm{S}$ & $\mathrm{N} / \mathrm{S}$ & $\mathrm{N} / \mathrm{S}$ \\
\hline Was there a control for co-interventions? & Yes & Yes & Yes \\
\hline Were con-interventions reported for each group separately? & Yes & Yes & Yes \\
\hline Was the patient blinded? & Yes & Yes & Yes \\
\hline Was the outcome assessor blinded? & Yes & Yes & Yes \\
\hline $\begin{array}{l}\text { Were drawback and dropout levels explained and appropriate? }(<20 \\
\% \text { short term },<30 \% \text { long term with no substantial bias }\end{array}$ & yes & Yes & Yes \\
\hline Was the instant of the result evaluation equivalent in groups? & Yes & Yes & Yes \\
\hline Did the analysis include an intention-to-treat analysis? & $\mathrm{N} / \mathrm{S}$ & $\mathrm{N} / \mathrm{S}$ & $\mathrm{N} / \mathrm{S}$ \\
\hline Was compliance acceptable in all groups? & Yes & Yes & Yes \\
\hline Was time of outcome assessment similar in all groups? & Yes & Yes & Yes \\
\hline Score: & $10 / 12$ & $10 / 12$ & $9 / 12$ \\
\hline
\end{tabular}




\section{Meta-analysis of all included RCTs :}

Results of the chosen studies are summarized in Table 4 , Table 5 and Table 6. Test of heterogeneity showed lower values $(\mathrm{p}$ - value $=0.4499)(\mathrm{p}$-value $=<0.5)$

TABLE (4) Number of success/other [healing, failure, or missing] in MTA and CEM pulpotomy groups at three follow-up periods; $\mathrm{O}=$ Other, $\mathrm{S}=$ Success

\begin{tabular}{|c|c|c|c|c|c|c|c|c|c|}
\hline Follow up & \multicolumn{2}{|c|}{ Months 6} & \multicolumn{3}{|c|}{ Months 12} & \multicolumn{4}{|c|}{ Months 24} \\
\hline$S_{m}$ & MTA & CEM & MTA & & CEM & MT & & $\mathrm{CE}$ & \\
\hline Grp. & $\mathrm{O} \quad \mathrm{S}$ & $\mathrm{O} \quad \mathrm{S}$ & $\mathrm{O}$ & $\mathrm{S}$ & $\mathrm{O} \quad \mathrm{S}$ & & $\mathrm{S}$ & & $\mathrm{S}$ \\
\hline Nosrat et al.2013 & $41 \quad 14$ & $35 \quad 18$ & 45 & 12 & $44 \quad 10$ & - & - & - & - \\
\hline Asgary and Eghbal 2013 & - & - & 155 & 12 & $170 \quad 9$ & - & - & - & - \\
\hline Malekafzaki et al. 2011 & 364 & 364 & 32 & 8 & $30 \quad 10$ & 34 & 6 & 32 & 8 \\
\hline
\end{tabular}

TABLE (5) Meta-analysis data summary of included studies

\begin{tabular}{|c|c|c|c|}
\hline & OR & $\begin{array}{c}\text { Lower } \\
\mathbf{9 5 \%}\end{array}$ & $\begin{array}{c}\text { Upper } \\
\mathbf{9 5 \%}\end{array}$ \\
\hline Nosrat et al. 2013 & 0.79 & 0.41 & 1.51 \\
\hline Asgary and Eghbal 2013 & 1.50 & 0.61 & 3.67 \\
\hline Malekafzali et al. 2011 & 0.64 & 0.20 & 2.11 \\
\hline
\end{tabular}

Mantel-Haenszel OR=0.92 95\% CI $(0.57,1.49)$

Test for heterogeneity $: X^{\wedge} 2(2)=1.72(p$-value $=0.4241)$

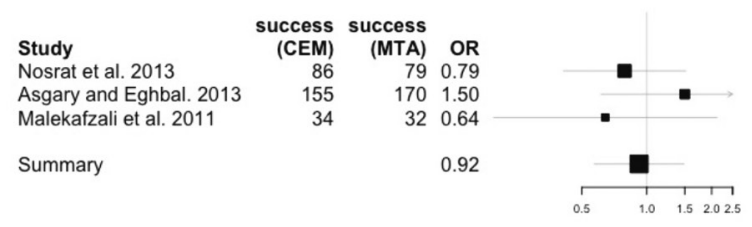

A

Study

Nosrat et al. 2013 Asgary and Eghbal. 2013 Malekafzali et al. 2011

Summary
TABLE (6) Meta-analysis data summary of included studies

\begin{tabular}{|c|c|c|c|}
\hline & RR & $\begin{array}{c}\text { Lower } \\
\mathbf{9 5 \%}\end{array}$ & $\begin{array}{c}\text { Upper } \\
\mathbf{9 5 \%}\end{array}$ \\
\hline Nosrat et al. 2013 & 0.85 & 0.55 & 1.32 \\
\hline Asgary and Eghbal 2013 & 1.46 & 0.63 & 3.38 \\
\hline Malekafzali et al.2011 & 0.71 & 0.28 & 1.81 \\
\hline
\end{tabular}

Mantel-Haenszel $R R=0.9495 \%$ CI $(0.66,1.35)$

Test for heterogeneity $: X^{\wedge} 2(2)=1.62(p-v a l u e=0.4455)$

$\begin{array}{lrrr} & \begin{array}{r}\text { success } \\ \text { Study }\end{array} & \text { success } \\ \text { (CEM) } & \text { (MTA) } & \text { OR } \\ \text { Nosrat et al. 2013 } & 86 & 79 & 0.79 \\ \text { Asgary and Eghbal. 2013 } & 155 & 170 & 1.50 \\ \text { Malekafzali et al. 2011 } & 34 & 32 & 0.64 \\ \text { Summary } & & & 0.92\end{array}$

B

Nosrat et al. 2013 Asgary and Eghbal. 2013 Malekatzali et al. 2011

Summary

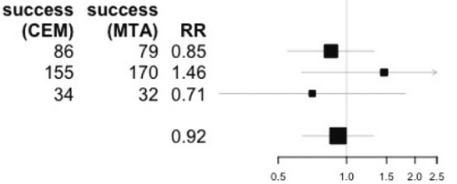

D

Fig. (1) Forest plot showing relative statistics of odds ratio (A and B) or relative risk ratio (C and D) between CEM and MTA for the selected studies profiled as a scaled horizontal axis. Vertical dashed axis represents "line of null effect." Black boxes represent point estimates for each study. Horizontal lines crossing boxes represent $95 \%$ confidence intervals of the study result. Right and left sides of the line of null effect represent MTA and CEM treatments, respectively. The summarized black box represents point estimate of the average of all 3 studies. 
Meta-analysis of the 3 input studies did not show any significant impact on the combined effect of both treatments. A slight improvement is directed toward (CEM) treatment. However, it is nonsignificant. (Figure 2 )

\section{DISCUSSION}

Meta-analysis is a reliable method that combines the results of many studies in a statistical manner ${ }^{(23)}$. This will offer a more accurate way for a specific treatment effect evaluation. This was the first systematic review to determine whether CEM or MTA has better outcomes for pulpotomy, as no agreement has been reached regarding the use of the two biomaterials. MTA has unique advantages and drawbacks. CEM has many favorable properties that foster its use as a pulpotomy agent. The obtained results revealed that there is no statistically significant difference in the rate of success among two treatment regimens. Final results demonstrated that both materials had similar clinical and radiographic success rates. However, CEM showed more favorable outcomes than MTA.

The SR search was limited to three RCTs. Because the outcome measures were binary, Mantel Haenszel method was used to calculate pooled $\mathrm{OR}$ and RR. There are different scales developed to assess and validate the quality of RCTs. In our analysis, we used the van Tulder list, which depends on randomization, concealment, blinding, outcome reporting and withdrawals and dropouts to evaluate the methodological quality of primary research ${ }^{\mathrm{x}}$. The van Tulder list is utilized by The Cochrane Collaboration Review Groups. In this metaanalysis, the included RCTs had a quality score more than eight; consequently, a meta-analysis of high-quality RCTs may demonstrate reliable results and conclusions.

Individually, the three studies included in the current meta-analysis support the use of both MTA and CEM. Malekafzaki et al. performed pulpotomy in primary molars using CEM or MTA in 40 children and checked clinical and radiographic evaluation at 6 months, 12 months, and 24 months after treatment. A total of 36,33 and 35 patients were available for 6-, 12- and 24-months follow-ups, respectively. After one year just one along with three teeth within the CEM and MTA groups acquired pathologic external root resorption, correspondingly. The resorbed teeth were then missed as a result of extraction/exfoliation in the 24-month follow-up; all other treated teeth were signed/symptom-free. Results showed that clinical and radiographic outcomes of both MTA/CEM groups were comparable at the follow-up periods without any significant differences ${ }^{(9)}$. Nosrat et al. treated 51 immature permanent molars with clinical carious exposure with symptomatic / asymptomatic pulpitis. Clinical and radiographic assessments were carried out at 6 and 12 months. Most open cases (49 teeth) exhibited pulp survival and an indication of ongoing root development after 12 months. Entire, complete apical closure (apexogenesis) took place in $76.8 \%$ and $73.8 \%$ of radiographically interpreted roots in CEM cement and MTA groups, correspondingly. There wasn't any significant difference regarding radiographic outcomes between two groups ${ }^{(10)}$. Asgary and Eghbal treated 413 permanent molars with acute irreversible pulpitis either MTA or CEM with follow-up at 12 months. There wasn't any significant variations in clinical $(p=0.7)$ and radiographic $(\mathrm{p}=0.4)$ success rates $^{(11)}$.

All three studies specified the procedure of pulpotomy. Clinical signs and radiography results are combined to determine the success or failure of the treatment. All studies reported the number of cases that lost to follow up. RCTs are regarded as the most reliable and accurate method for experimental design. Also, RCTs are occupying a high rank in the hierarchy of quality of evidence and can produce the most accepting causal relationship other than other clinical studies such as cohort, cross-sectional, or case control studies. All the included studies 
were described as randomized. Nosrat et al. used the computer-generated permuted blocks; where each block contains four teeth. The allocation of participants and the implementation were in the dental center to ensure concealment ${ }^{(10)}$. Asgary and Eghbal used computer-based randomization schedule ${ }^{(11)}$. However, Malekafzaki et al. failed to describe their method of randomization clearly ${ }^{(9)}$. But, at the same time, they used the split mouth design which is proved to be an efficient layout. Ensuring the evaluator is blind to treatment variables when assessing outcomes is important. All studies failed to state that reviewers are blind to treatment method. Practitioners were blind to the materials when they were performing the pulpotomies because the characteristics two materials are water based pulp capping materials. As to the sample size, the three papers reported rationale for the sample size. The importance of sample size calculation cannot be underestimated. A small sample size will lead to a lower power of the test and lack of adequate evidence, whereas a larger one will cause the difficulties of follow-up control and a waste of additional resources such as labor, money and time. Meta-analysis showed homogeneity ( $\mathrm{p}$-value $=0.4499$ ), which is considered to be a high point for the included studies. Studies conducted by Malekafzaki et al. and Nosrat et al. were performed in teaching hospital schools. Trials displayed by Asgary and Eghbal were done by dentists working in multi dental primary health care centers which made extrapolation for general practitioners easier after been trained in the center.

Publication bias is considered to be the main obstacle in reporting RCTs. It is defined as "the tendency of journals' reviewer/editors to accept RCTs for publication based on the direction/strength of the findings" ${ }^{(25)}$. Publication bias might be due to the denial of some authors to publish negative results of performed RCTs. That is why results of systematic reviews/meta-analyses depended only on published RCTs only might be deceiving. It is recognized that approximately 25 studies are required to calculate the publication bias ${ }^{(25)}$. Since only three studies were included in the review publication bias was neither confirmed nor denied. Moreover, all of the included RCTs (3 out of 3 ) did not show any statistical significance in their results.

The current systematic review of the trials with meta-analysis displayed that based on available information, the results demonstrated the statistically insignificant difference in success rate between the two treatment protocols. On the other hand, CEM performs superiorly to MTA as a material used in pulpotomy. However, larger, blind, RCTs are needed to be conducted to provide more reliable clinical evidence.

\section{CONCLUSION}

Concerning the validity and homogeneity of the RCTs, the results displayed superior treatment outcome of CEM pulpotomy in human primary and permanent teeth in comparison to those treated with MTA, although it was insignificant.

\section{REFERENCES}

1. McDonald RE, Avery DR, Dean JA. Treatment of deep caries, vital pulp exposure and pulpless teeth. Dentistry for the child and adolescent. In: McDonald RE, Avery DR; 8th ed. St. Louis, Mo: Mosby Co; 2004. p 389-412.

2. Hasselgren G, Reit C. Emergency pulpotomy: pain relieving effect with and without the use of sedative dressings. J Endod. 1989; 15:254-256.

3. Bender IB. Reversible and irreversible painful pulpitis: diagnosis and treatment. Aust Endod J. 2000;26:10-14.

4. Fallahinejad Ghajari M, Mirkarimi M, Vatanpour M, Kharrazi Fard MJ. Comparison of pulpotomy with formocresol and MTA in primary molars: a systematic review and metaanalysis. Iran Endod J .2008;3:45-49.

5. Barrieshi-Nusair K, Qudeimat MA. A prospective clinical study of mineral trioxide aggregate for partial pulpotomy in cariously exposed permanent teeth. $J$ Endod.2006;32: 731-735.

6. Aeinehchi M, Eslami B, Ghanbariha M, Saffar AS. Mineral trioxide aggregate (MTA) and calcium hydroxide as pulpcapping agents in human teeth: a preliminary report. Int Endod J .2003;36:225-231. 
7. Witherspoon DE, Small JC, Harris GZ. Mineral trioxide aggregate pulpotomies: a case series outcomes assessment. $J$ Am Dent Assoc. 2006;137:610-618.

8. Eghbal MJ, Asgary S, Baglue RA, Parirokh M, Ghoddusi J. MTA pulpotomy of human permanent molars with irreversible pulpitis. Aust Endod J. 2009;35:4-8.

9. Malekafzali B, Shekarchi F, Asgary S. Treatment outcomes of pulpotomy in primary molars using two endodontic bioma- terials. A 2-year randomised clinical trial. Eur $J$ Paediatr Dent . 2011;12:189-93.

10. Nosrat A, Seifi A, Asgary S.Pulpotomy in caries-exposed immature permanent molars using calcium-enriched mixture cement or mineral trioxide aggregate: a randomized clinical trial. Int J Paediatr Dent. 2013; 23: 56-63.

11. Asgary s and Eghbal M. Treatment outcomes of pulpotomy in permanent molars with irreversible pulpitis using biomaterials: A multi-center randomized controlled trial .Acta Odontologica Scandinavica. 2013; 71: 130-136.

12. Dominguez MS, Witherspoon DE, Gutmann JL, Opperman LA. Histological and scanning electron microscopy assessment of various vital pulp-therapy materials. $J$ Endod. 2003;29:324-33.

13. Asgary S, Parirokh M, Eghbal MJ, Ghoddusi J, Eskandarizadeh A. SEM evaluation of neodentinal bridging after direct pulp protection with mineral trioxide aggregate. Aust Endod J. 2006;32:26-30.

14. Asgary S, Eghbal MJ, Parirokh M, Ghanavati F, Rahimi H. A comparative study of histologic response to different pulp capping materials and a novel endodontic cement. Oral Surg Oral Med Oral Pathol Oral Radiol Endod. 2008; 106:609-614.

15. Asgary S, Ehsani S. Permanent molar pulpotomy with a new endodontic cement: a case series. J Conserv Dent. 2009; 12: 31-36.

16. Asgary S. Calcium-enriched mixture pulpotomy of a human permanent molar with irreversible pulpitis and condensing apical periodontitis. J Conserv Dent. 2011; 14:90-93.

17. Asgary S, Eghbal MJ. The effect of pulpotomy using a calcium-enriched mixture cement versus one-visit root canal therapy on postoperative pain relief in irreversible pulpitis: a randomized clinical trial. Odontology. 2010;98: 126-133.

18. Asgary S, Shahabi S, Jafarzadeh T, Amini S, Kheirieh S. The properties of a new endodontic material. J Endod. 2008;34: 990-993.

19. Asgary S, Eghbal MJ, Parirokh M. Sealing ability of a novel endodontic cement as a root-end filling material. J Biomed Mater Res. 2008;87:706-709.

20. Egger M, Smith G, Phillips A. Meta-Analysis: principles and procedures. Brit Med J. 1997;315:1533-1537.

21. Liberati A, Altman DG, Tetzlaff J, Mulrow C, Gotzsche PC, Ioannidis JPA, Clarke M, Devereaux PJ, Kleijnen J, Moher D. The PRISMA Statement for Reporting Systematic Reviews and Meta-Analyses of Studies that Evaluate Health Care Interventions: Explanation and Elaboration. PLoS Medicine 2009; 6:1-28.

22. Moher D, Liberati A, Tetzlaff J, Altman DG, The PRISMA Group (2009). Preferred Reporting Items for Systematic Reviews and Meta-Analyses: The PRISMA Statement. PLoS Med 6(7): e1000097. doi:10.1371/journal. pmed1000097.

23. van Tulder M, Furlan A, Bombardier C, Bouter L. Updated method guidelines for systematic reviews in the Cochrane Collaboration Back Review Group. Spine (Phila Pa 1976) 2003;28:1290-1299.

24. Shirvani A, Hassanizadeh R, Asgary S. Mineral Trioxide Aggregate vs. Calcium Hydroxide in Primary Molar Pulpotomy: A Systematic Review . Iranian Endodontic Journal. 2014;9:83-88.

25. Glasziou P (2001) Systematic Reviews in Health Care: a Practical Guide. Cambridge, New York: Cambridge University Press. 